\title{
Low Temperature Magneto-Optical Kerr Effect Experimental System with a Cryogen-Free Sample Environment
}

\section{Chidubem A. Nwokoye ${ }^{1,2 *}$, Lawrence H. Bennett ${ }^{1}$, Edward Della Torre ${ }^{1}$, Abid Siddique', Frank A. Narducci' ${ }^{2}$, Mohammadreza Ghahremani' and Khurram S. Khattak'}

\author{
${ }^{1}$ Institute for Magnetic Research, Department of Electrical and Computer Engineering, The George Washington \\ University, USA
}

${ }^{2}$ Naval Air Systems Command, Avionics, Sensors and E*Warfare Department, USA

\begin{abstract}
Interest in the study of ferromagnetism in nanostructures at low temperatures is growing. We report the development of a low temperature MOKE experimental system with a cryogen-free sample environment, permitting magneto-optical measurements down to $9 \mathrm{~K}$. We report how the experimental system is utilized to study the temperature variation of the magneto-optical (Kerr rotation angle) property of a Co/Pd multilayered film.
\end{abstract}

\section{Keywords}

Magneto-Optical Kerr Effect (MOKE), Magneto-optical, Cryogen-free, Hysteresis

\section{Introduction}

The magneto-optical Kerr effect (MOKE), discovered by Rev. John Kerr [1-3] in 1877, provides insight into the complex rotation of the polarization plane of linearly polarized incident light reflected from the surface of a magnetic material. The complex rotation angle, named after its founder, is known as the Kerr angle which comprises of the Kerr rotation $\theta_{K}$ and Kerr ellipticity $\varepsilon_{K}$ as real and imaginary parts respectively. Early demonstration of the effect was conducted on iron $(\mathrm{Fe})$ and gadolinium-cobalt (Gd-Co) films [1-6] which showed how both the Kerr rotation and Kerr ellipticity exhibit hysteresis and the sensitivity of the hysteresis loops varied as a function of film thickness and temperature. Recently, MOKE has been applied for imaging magnetic domains $[7,8]$, in-plane magnetization measurements [9], surface stress measurements [10], and studying materials for highdensity magneto-optic information and storage media [11,12].

Research in low temperature physics of magnetism in ferromagnetic materials is gaining attention. Experimental work, by Bennett and Della Torre [13], on Bose-Einstein condensation in metallic nanostructures observed from a variety of magnetic signatures indicates the importance of research in ferromagnetism at low temperatures. Optical experiments have been performed at both liquid nitrogen and liquid helium temperatures via sample immersion in liquid cryogen cryostat systems [10,14-19]. Optical experiments using liquid helium cryostats are complex and expensive due to the use of liquid helium as the direct cooling medium. This presents a principal issue of distortion and scattering of light by cryogen bubbles and schlieren (optical inhomogeneities in transparent material not necessarily visible to the human eye) [20]. Qiu et al. [21], reports in a surface MOKE system, the dependence of index of refractions of initial and final media to magneto-optical (MO) properties of multilayered magnetic thin films. Thus, less accuracy in MO properties is introduced in liquid helium cryostat systems due to the additional index of refraction from liquid helium [22] in the optical travel path. In the current paper, we describe a MOKE experimental setup with a cryogen-free sample environment designed to study $\mathrm{MO}$ properties of ferromagnetic thin film materials at temperatures as low as $9 \mathrm{~K}$.

The next section of this paper provides an overview of the experimental setup. It is followed by a description of the experiment procedure. The results section presents some experimental Kerr loops which illustrate the MOKE results at room temperature and at cryogenic temperatures. The conclusion section presents a summary of the work.

\section{Experiment}

\section{Setup}

We designed and built an in-situ experimental system, which consists of an automated photoelastic modulator (PEM)-based MOKE apparatus [6] and closed cycle refrigeration (CCR) system for the measurement of $\mathrm{MO}$ properties of thin film materials at

*Corresponding author: Chidubem A. Nwokoye, Institute for Magnetic Research, Department of Electrical and Computer Engineering, The George Washington University, Washington and Naval Air Systems Command, Avionics, Sensors and E*Warfare Department, Patuxent River, MD 20670, USA, E-mail: dubem@gwmail.gwu.edu

Received: July 27, 2015: Accepted: August 22, 2015: Published: August 26, 2015

Copyright: (C) 2015 Nwokoye CA. This is an open-access article distributed under the terms of the Creative Commons Attribution License, which permits unrestricted use, distribution, and reproduction in any medium, provided the original author and source are credited. 


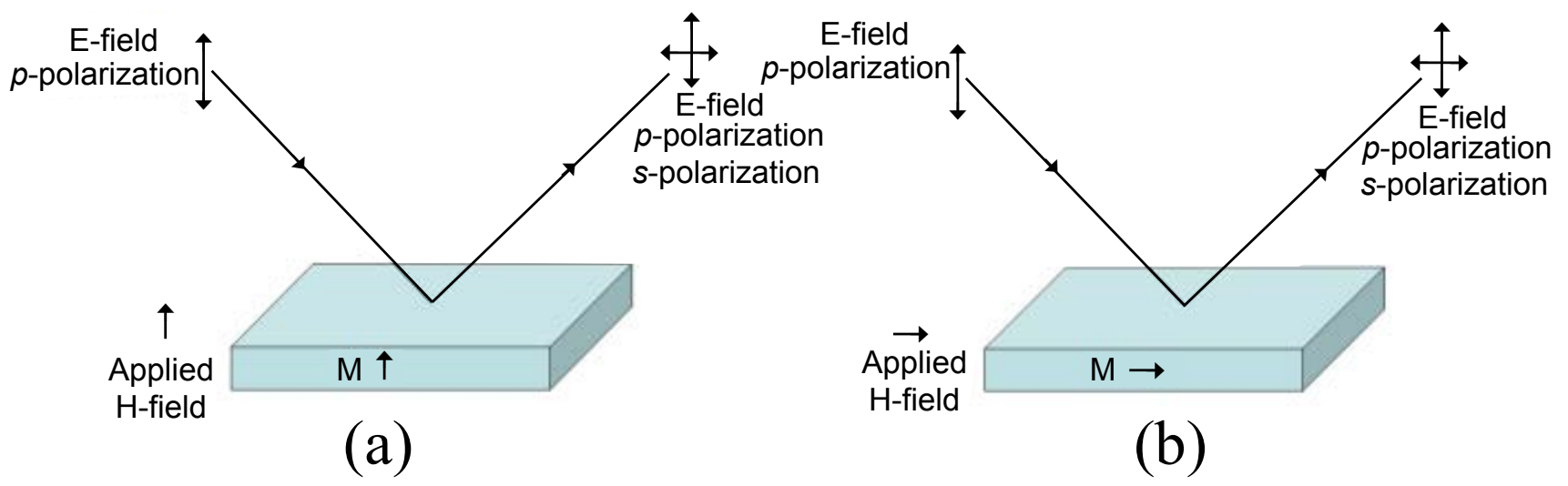

Figure 1: Illustration of mode configurations for the MOKE apparatus (a) polar (b) longitudinal.

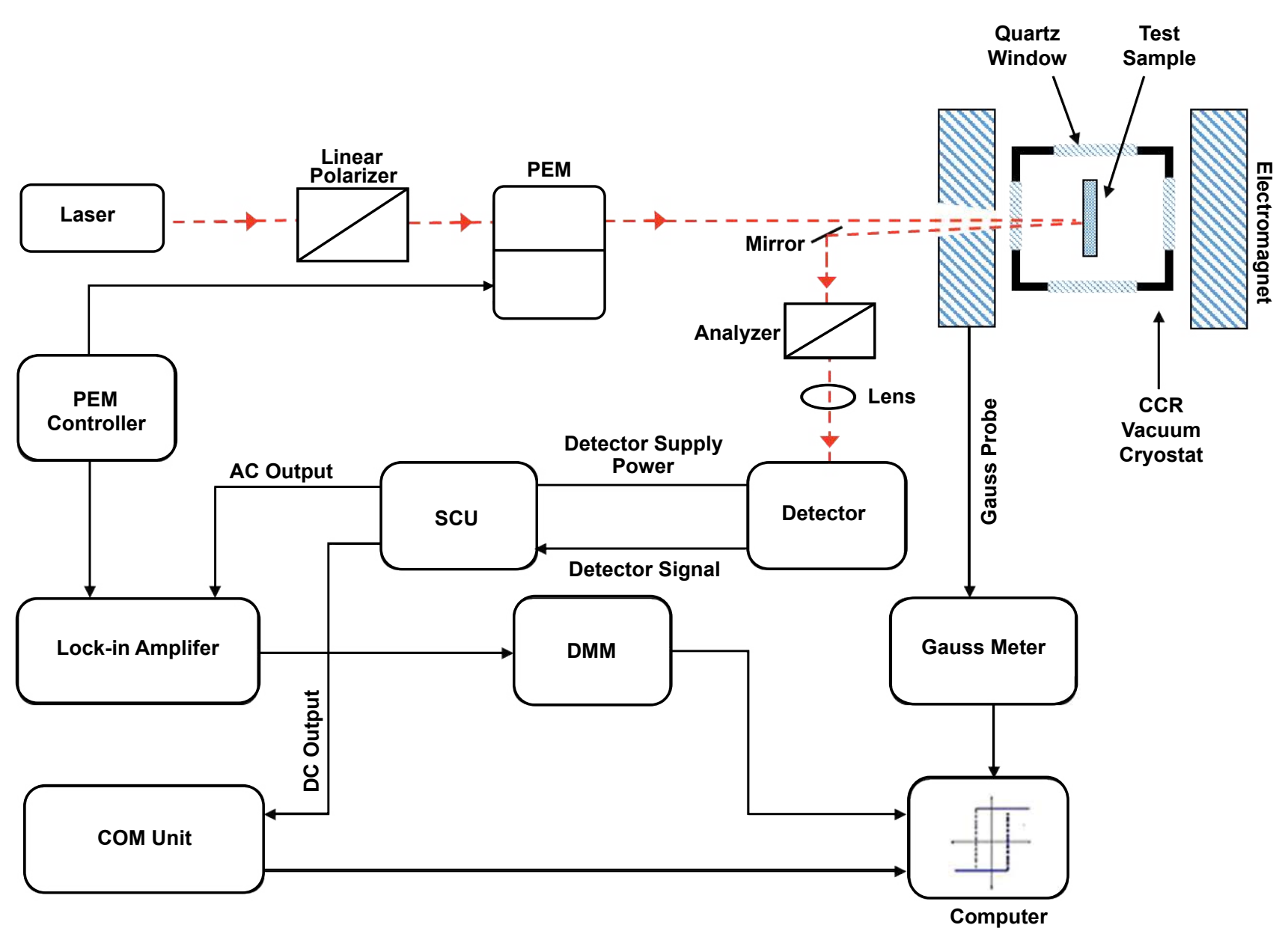

Figure 2: Schematic top-view layout of the in-situ experimental setup that consists of a PEM-based MOKE apparatus with CCR Vacuum Cryostat.

temperatures from $9 \mathrm{~K}$ to $325 \mathrm{~K}$. The CCR system, which comprises of a cold-head (two-stage cryogenic refrigerators (model CH-204FF, SHI Cryogenics Group), with cooling power specification of 7.1 watts at $20 \mathrm{~K}$ (second stage), 16.2 watts at $80 \mathrm{~K}$ (first stage)), vacuum cryostat (VC) (by Janis Research), helium compressor (model HC4E, SHI Cryogenics Group) and temperature controller (model 336, Lake Shore Cryotronics), provides a convenient means of cooling a test sample to temperatures as low as $9 \mathrm{~K}$ and it is designed to be compatible with a MOKE apparatus. The system operates based on the Gifford-McMahon (G-M) thermodynamic cycle [23] via compression and expansion of helium gas in a closed loop circulation. Thus, feasibility of studying magneto-optical properties of magnetic samples at low temperatures can be achieved.

The schematic layout of the in-situ experimental system setup is shown in figure 1 and 2. The MOKE apparatus can be set to operate in either polar or longitudinal modes (Figure 1). In polar and longitudinal Kerr effects, the reflected light experiences both Kerr rotation and Kerr ellipticity. Components of the MOKE consists of the following: a helium-neon laser source $(5 \mathrm{~mW}, 632.8 \mathrm{~nm}$ wavelength with a $2 \mathrm{~mm}$ beam diameter), linear polarizer (polarizing prism), PEM crystal, PEM controller, electromagnet (maximum field of $0.8 \mathrm{~T}$ powered by bipolar power supply), mirror, lens, analyzer (polarizing prism), signal conditioning unit (SCU), lock-in amplifier, COM Unit, 
digital multi-meter (DMM), gauss probe, gauss meter and a detector (silicon photovoltaic diode). Parts of the MOKE apparatus (COM unit, the DMM and the Gauss meter) are connected to a computer via GPIB cables and PCI interface; the data acquisition and measurement protocols are controlled by a Visual Basic program.

The light from the laser source propagates through the linear polarizer and PEM crystal and undergoes a linear polarization and retardation with a modulation (between right hand and left hand circular polarizations with intermediate linear/elliptical states) frequency of $f=57 \mathrm{kHz}$ before reflecting off the sample inside the cryostat. The amplitude of the modulated retardation is kept constant at $\pi / 2$ over the whole wavelength region, by setting the in-built wavelength-proportional voltage of the modulator controller. The angle of incidence to the sample surface is approximately 2 degrees, which is near-perpendicular to the surface of the sample. The size of the hole in the electromagnet pole cap is half an inch in diameter and 11 inches in length, and therefore restricts the maximum angle difference between the incident and reflected laser to be approximately 2 degrees for polar mode configuration. Larger incident angles can be studied but it will involve having a mirror between the electromagnet poles to collect the reflected laser. The reflected light propagates through the analyzer and is focused on the detector by a lens. The received signal from the detector is sent into the signal conditioning unit which splits the signal into its broadband AC and lowpass DC signals, amplifies these signals, then applies the amplified signals to AC and DC outputs. The SCU's AC and DC outputs are connected to the lock-in amplifier and the COM unit respectively. The lock-in amplifier receives a reference signal ( $f$ or $2 f$ ) from the PEM controller and selects the AC signals with either $f$ or $2 f$ frequency in the AC signals from the SCU. The signal-to-noise ratio of the selected AC signal is increased and sent to the DMM. The DMM measures and displays the AC signal voltage magnitude before it sends the digitized amplitude values to the computer. The COM Unit digitizes the DC signal from the SCU and sends it to the computer for data acquisition processing. The intensity ratio of the AC signal to DC signal is derived [6] (for $0^{\circ}$ analyzer angle and small Kerr rotation angles) to be

$$
\begin{aligned}
& I_{f} / I_{d c} \approx 4 A J_{1}\left(\delta_{0}\right) \varepsilon_{K}, \text { and } \\
& I_{2 f} / I_{d c} \approx 4 B J_{2}\left(\delta_{0}\right) \theta_{K},
\end{aligned}
$$

where $J_{n}(x)$ is an $\mathrm{n}$-th order Bessel function. Equation (1) and (2) show a linear relationship of the Kerr rotation $\theta_{K}$ and Kerr ellipticity $\varepsilon_{K}$ with detected $I_{f}$ and $I_{2 f}$ laser intensities respectively. The reference signal input channel of the lock-in amplifier used in the setup has a frequency band of $0.5 \mathrm{kHz}$ to $100 \mathrm{kHz}$, thus, the setup is only capable of selecting either $1 f$ or $2 f$ on an individual basis. For measurement of the Kerr rotation in the setup, we connected $2 f$ output port of the PEM controller to the reference signal input of the lock-in amplifier. Selecting the $1 f$ output will result in the measurement of the Kerr ellipticity. The data processing software on the computer computes the intensity ratios. The magneto-optical properties $\left(\theta_{K}\right.$ and $\left.\varepsilon_{K}\right)$ are directly proportional to the intensity and the coefficients in Equations 1 and 2 can be determined by calibration procedure in [6].

The cryostat unit of the CCR system, shown in figure 3, comprises an aluminum cube-like shroud with four O-ring sealed quartz windows for optical access to the test sample, a cylindrical aluminum radiation shield with optical access, a sample mount, a sample holder and a cold finger rod equipped with a $50 \mathrm{ohms}$ cartridge heater and a GaAIAs diode temperature sensor for precise temperature control via the temperature controller. The temperature controller is equipped with a temperature set-point option, a manual open-loop heater control option, and a closed-loop heater control option. The

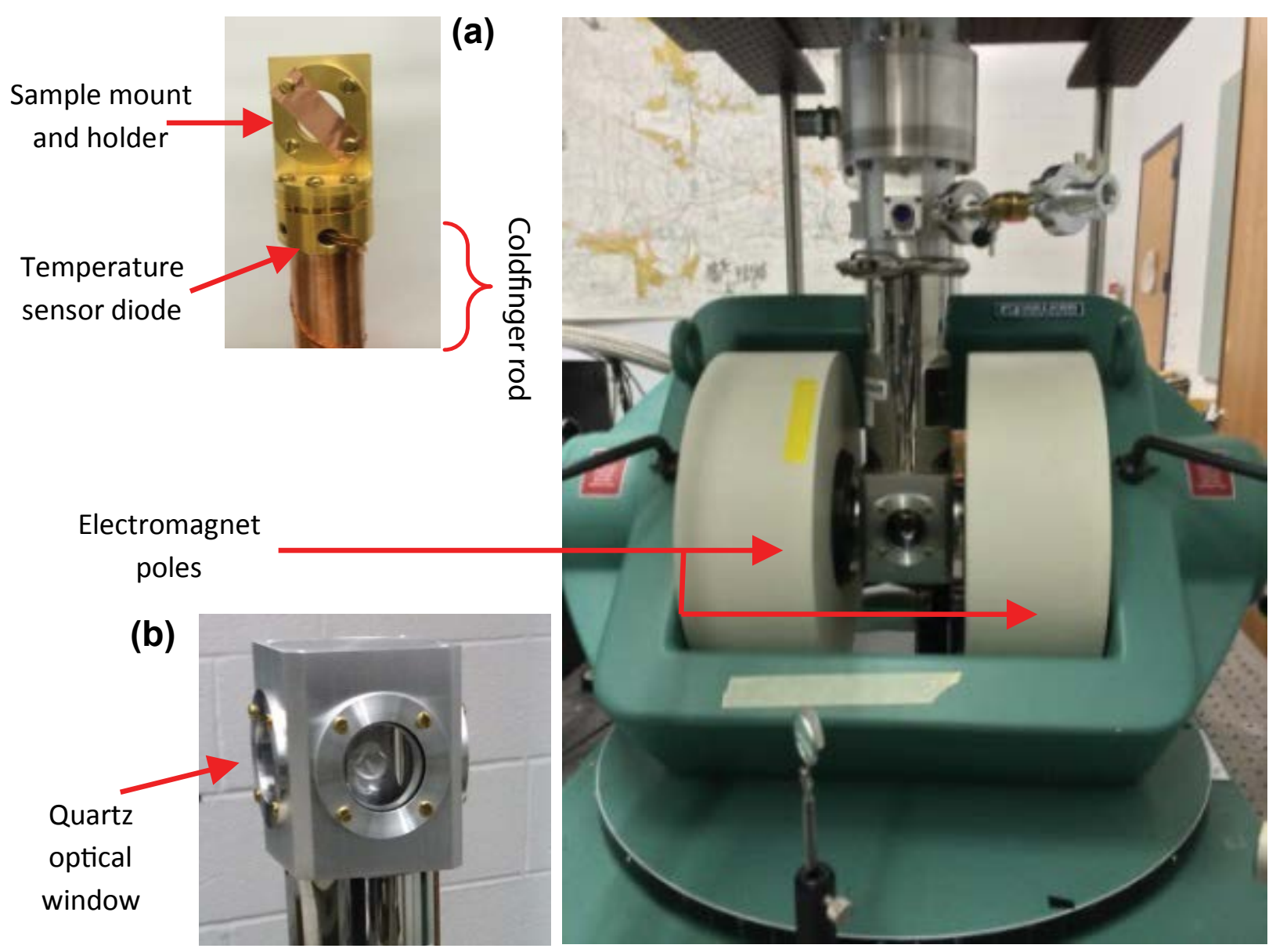

(c)

Figure 3: (a) Sample mount and holder inside cryostat (b) Aluminum Shroud of cryostat (c) Cryostat positioned between electromagnet. 
closed-loop heater control option uses the PID control algorithm. The control equation for the PID algorithm has three variable terms: proportional $(P)$, integral $(I)$, and derivative $(D)$ as

$$
\text { Heater Output } t_{(\text {closed-loop })}=P\left(e+I \int(e) d t+D \frac{d e}{d t}\right),
$$

Where the error $e=$ set-point - feedback reading. For a given temperature set-point, the optimal tuning procedure for obtaining the proportional, integral and derivative values are as follows, (a) Set the $I$ and $D$ values to zero and gradually start with a small value for $P$ until oscillation in the temperature reading begins about the set-point temperature, then obtain an estimate of the period of the oscillation, $t_{p}$. (b) Set the integral value to the value of the ratio of 1000 to estimated period of oscillation $\left(I=1000 / t_{p}\right)$. (c) A zero derivative setting is recommended when the control system is rarely changed but a small value can work to boost the heater output when the set-point changes quickly.

Any desired temperature within the appropriate operation range can be set and the settings of the PID parameters controls the temperature stability and response time of the desired temperature. In general, when operating in the lowest temperatures regime $(<70 \mathrm{~K}$, where the heat capacities are smallest), the $P$ value should be low, the $I$ value should be high, and the $D$ value can remain zero throughout the operating range. Likewise, for higher temperature set-points, the $P$ value should be high and the $I$ value should be low. For example, after cooling the cryostat to its lowest temperature and entering a set-point temperature of $20 \mathrm{~K}$ and setting the PID values to $P=20, I=50, D=$ 0 , the observed temperature reading of $20 \mathrm{~K}$ was constant within \pm 1 $\mathrm{K}$ change for a duration of approximately 14 minutes.

During cooldown, the radiation shield which mounts to the firststage of the cold-head, intercepts the room temperature radiation before it reaches the sample, allowing the lowest possible sample temperature to be achieved. The cryostat unit is positioned between the two electromagnets and carefully adjusted so that the incident and reflection laser beam from the sample can directly pass through a small hole in the pole cap of an electromagnet; hence, both the incident and the reflection laser beams are perpendicular to the surface of the sample.

\section{Procedure}

An experimental procedure was used in the system setup to systematically measure the MO properties of thin ferromagnetic films at temperatures below $70 \mathrm{~K}$ (liquid nitrogen temperature). We illustrated this with a $\mathrm{Co} / \mathrm{Pd}$ multilayered thin film. Firstly, a thermally conductive adhesive was applied on the substrate face of the $\mathrm{Co} / \mathrm{Pd}$ sample, opposite side of structure shown in figure $4 \mathrm{a}$, and glued to a rectangular copper sheet (Figure 4b).

For this experiment, we used the thermally conductive epoxy Stycast $2850 \mathrm{FT} / 24 \mathrm{VL}$ (by Henkel Loctite) which has a thermal conductivity of $0.8 \mathrm{~W} /(\mathrm{m} . \mathrm{K})$ at about $77 \mathrm{~K}$ and $1.02 \mathrm{~W} /(\mathrm{m} . \mathrm{K})$ at 208 $\mathrm{K}$ to $378 \mathrm{~K}$ (values at other temperatures was not available at time of the experiment). Other alternative thermal conductive adhesives that are applicable are: Apiezon N grease (by M\&I Materials Ltd), Krytox grease (by Chemours company), and Crycon grease (by Janis Research company). Both ends of the copper sheet are screwed to the sample mount (Figure 3a) supported by the sample holder and cold finger rod in the cryostat unit. The sample is known to have perpendicular magnetization; hence, for optimum magnetic response, the MOKE apparatus is set to polar mode. The cryostat unit was evacuated to a vacuum pressure of $1 \times 10^{-4}$ torr (manufacturer's specified value) or less via a turbo-molecular vacuum pump. Better vacuum pressure levels are essential to provide greater thermal insulation, resulting in shorter cooldown times and lower final temperatures. Outgassing and O-ring permeation causes the pressure level in the cryostat to slowly rise over time. We periodically re-evacuated the cryostat during the experiment to maintain the pressure level below $1.0 \times 10^{-4}$ torr. The

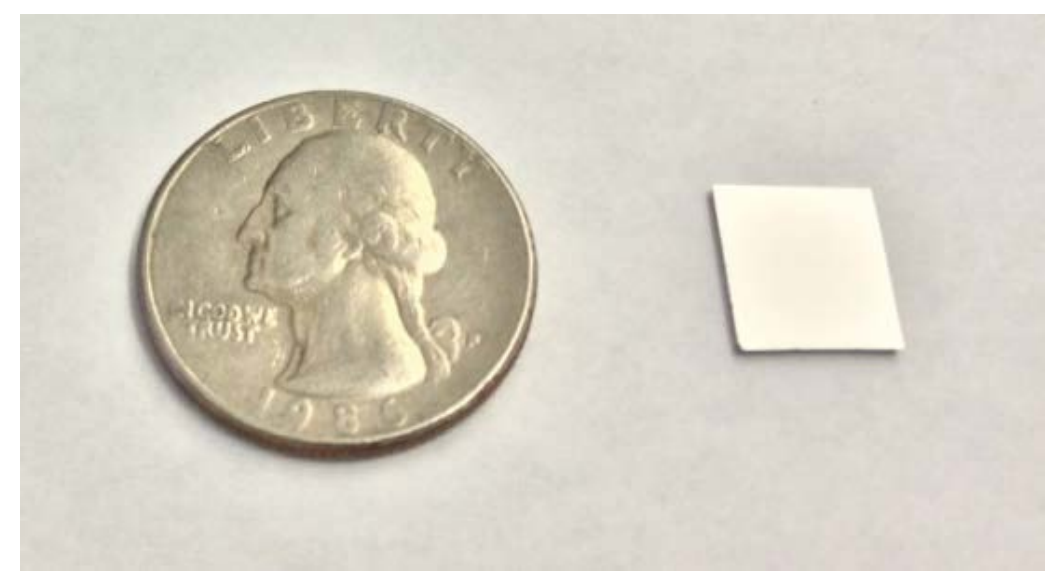

(a)

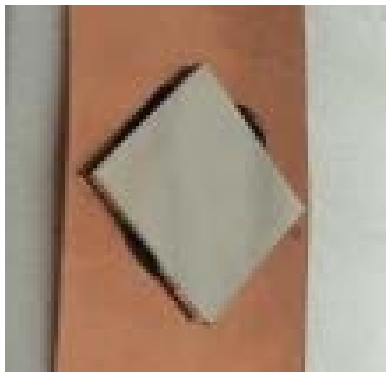

(b)

Figure 4: (a) Picture of Co/Pd test sample (b) Co/Pd test sample glued with stycast epoxy onto a Cu sheet for mounting on sample holder in cryostat 


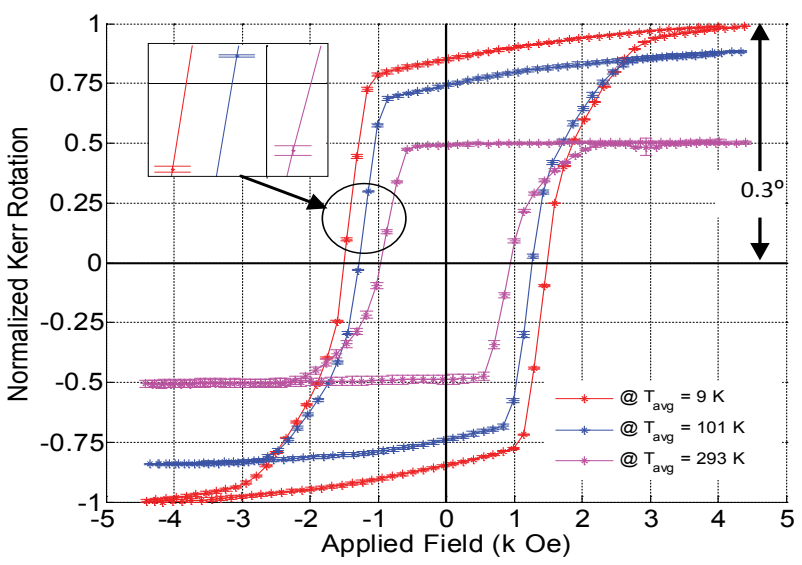

Figure 5: Normalized Kerr rotation $\theta_{K}$ major hysteresis loop at $9 \mathrm{~K}, 101 \mathrm{~K}$ and $293 \mathrm{~K}$ for Co/Pd thin film sample. The inset shows error bar depicting standard error for the examined temperatures respectively.

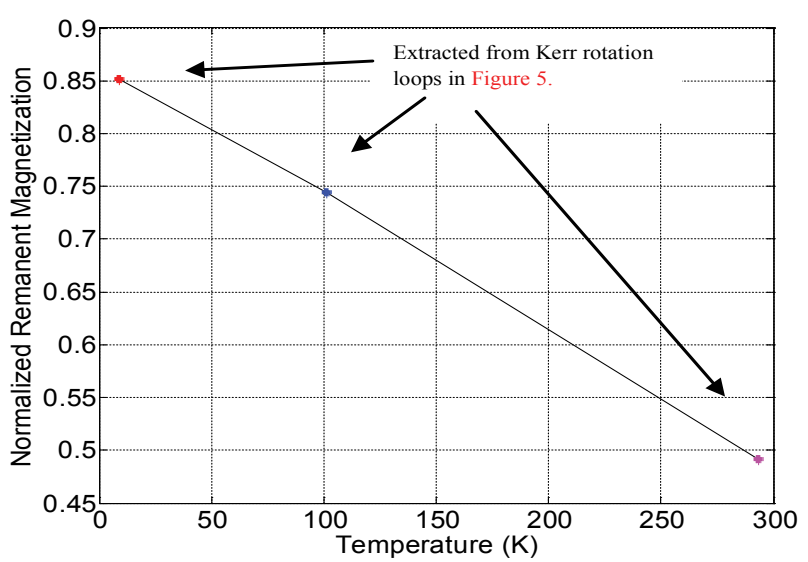

Figure 6: Extracted normalized remanent magnetization (Kerr rotation at zero field) response with temperature for Co/Pd thin film sample.

temperature controller was switched on and calibrated with the GaAIAs diode temperature sensor inside the cryostat unit to measure and record the temperature of the sample. Prior to cooldown, to mitigate the effect sample contamination and heat conduction from residual gasses to the sample, the temperature controller which controls the heater and monitors the temperature sensor, was set to a temperature above room temperature $(\sim 305 \mathrm{~K})$ for about 40 minutes of operation. This causes contaminants such as water vapor and other condensable gases that can freeze at cryogenic temperatures to settle on the surface of the radiation shield rather than on the sample.

Secondly, standard MOKE calibration procedure [6] was followed to eliminate excessive noise and distortion of MO hysteresis loop. Polar MO hysteresis loops for either or both Kerr rotation $\theta_{K}$ and Kerr ellipticity $\varepsilon_{K}$ were examined at $632.8 \mathrm{~nm}$ with the automated PEMbased MOKE system. The signals in the detector that correspond to the fundamental frequency, $1 f$ (proportional to $\varepsilon_{K}$ ) or $2 f$ (proportional to $\theta_{K}$ ) of the PEM modulation frequency was measured while sweeping the magnetic field $(4.3 \mathrm{kOe}$ to $-4.3 \mathrm{kOe})$ from positive to negative saturation and back again. Three MO hysteresis loops were obtained at room temperature and averaged. Thereafter, critical magnetic parameters such as coercivity and remanent magnetization were extracted from the averaged loop and recorded.

Thirdly, the compressor was switched on to initiate the cooling of the sample based on the G-M thermodynamic cycle. During the expansion phase of the cycle, heat is removed from the cold finger rod, on which the sample mount and holder are mounted. The expanded helium gas in the cold head unit returns back to the compressor

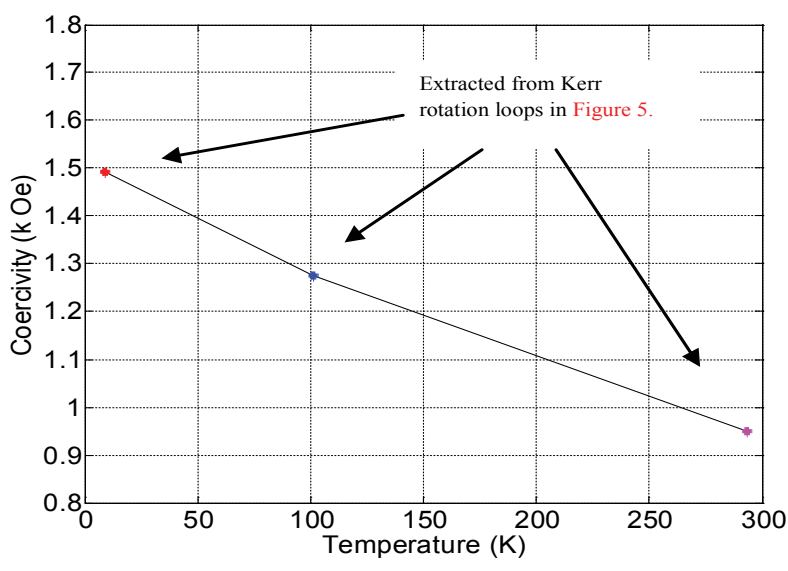

Figure 7: Coercivity response with temperature extracted from measured Kerr rotation loops shown in figure 5 .

and gets compressed and the process is continuously repeated until the lowest temperature is attained. Sample cooling is attained by conduction via the copper-based cold finger rod and maintained by the high vacuum levels inside the cryostat unit. The temperature of the sample increases by less than $1 \mathrm{~K}$ by the illumination of the laser.

MO hysteresis loops were measured and recorded when the sample temperature reached $9 \mathrm{~K}$. The heater was then set to slightly raise the sample temperature to attain a user defined higher temperature, and MO hysteresis loops were measured and recorded. The process can be repeated for any desired temperature in the $9 \mathrm{~K}$ to $325 \mathrm{~K}$ range. For illustration, we report results at approximate temperatures of 9 $\mathrm{K}, 101 \mathrm{~K}$ and $293 \mathrm{~K}$.

\section{Experimental Results}

The averaged Kerr rotation major hysteresis loop at $9 \mathrm{~K}, 101 \mathrm{~K}$ and $293 \mathrm{~K}$ for the Co/Pd test sample are shown in figure 5. The averaged loops at each reported temperature gave a maximum standard error percentage of $0.081,0.098$ and 0.283 respectively. A significant increase in the area of the hysteresis loop, which is proportional to energy dissipation, was observed as the temperature decreased. The loops at $9 \mathrm{~K}$ and $101 \mathrm{~K}$ showed changes in Kerr rotation as the applied field decreased from saturation to zero while the loop at 293 $\mathrm{K}$ showed a negligible change, thus, indicating that the relationship between Kerr rotation and structural readjustment in the sample is more evident at lower temperatures.

The increase in the area of hysteresis loops leads to the observation of an increase in nucleation field (applied field needed for occurrence of nucleation of opposite oriented domains in the sample) as the temperature decreases from $293 \mathrm{~K}$ to $9 \mathrm{~K}$. Significant increase in Kerr rotation at zero field, which directly relates to the remanent magnetization, was also observed as the temperature decreases. Figure 6 depicts this significant change in remanent magnetization with temperature.

The coercivity, which is the applied field that results to a zero Kerr rotation, was found to increase with decrease in temperature. This effect was anticipated based on Sharrock's law for remanence coercivity dependence on temperature [24]. Figure 7 depicts the extracted coercivity from the Kerr rotation loops.

\section{Conclusion}

A low temperature experimental system, which consists of an automated PEM-based MOKE apparatus and CCR system with a cryogen-free sample environment for the measurement of surface magnetization, is reported. A Kerr rotation hysteresis loop at 9 $\mathrm{K}$ was obtained and when compared with measured data at $293 \mathrm{~K}$ (approximately room temperature), significant decrease in the area 
of the hysteresis loop was observed including a decrease in remanent magnetization, coercivity, and dependency of structural readjustment with magnetization.

\section{Acknowledgements}

The research at The George Washington University was supported in part by the Naval Air Systems Command's NISE Section 219 funds. The author will like to thank members of the IMR group for their valuable discussion during the course of the research.

\section{References}

1. J Kerr (1877) XLIII. On rotation of the plane of polarization by reflection from the pole of a magnet. Philos Mag 3: 321-343

2. J Kerr (1878) XXIV. On reflection of polarized light from the equatorial surface of a magnet. Philos Mag 5: 161-177

3. Weinberger $P$ (2008) John Kerr and his effects found in 1877 and 1878 Philos Mag Lett 88: 897-907.

4. Moog ER, Bader SD(1985) Smoke signals from ferromagnetic monolayers $p(1 \times 1) \mathrm{Fe} / \mathrm{Au}(100)$. Superlattices Microstruct 1: 543-552.

5. Bader SD, Moog ER, Grunberg P (1986) Magnetic hysteresis of epitaxiallydeposited iron in the monolayer range: A Kerr effect experiment in surface magnetism. J Magn Magn Mater 53: 295-298.

6. K. Sato (1981) Measurement of Magneto-Optical Kerr Effect Using PiezoBirefringent Modulator. Jpn J Appl Phys 20: 2403-2409.

7. Fry RA, Bennett LH, Della Torre E (1999) Kerr imaging of a Co/Pt bimodal magneto-optical medium J Appl Phys 85: 5169-5171.

8. Mallick S, Bedanta S, Seki T, Takanashi K (2014) Magnetic domain imaging in L10 ordered FePt thin films with in-plane uniaxial magnetic anisotropy. J Appl Phys 116: 133904-6.

9. HalahovetsY, SiffalovicP, Jergel M, Senderak R, Majkova E, et al. (2011) Scanning magneto-optical Kerr microscope with auto-balanced detection scheme. Rev Sci Instrum 82: 083706-6.

10. Premper J, Sander D, Kirschner J (2012) A combined surface stress and magneto-optical Kerr effect measurement setup for temperatures down to 30 $\mathrm{K}$ and in fields of up to $0.7 \mathrm{~T}$. Rev Sci Instrum 83: 073904-8.
11. Klahn S, Hansen P, Greidanus FJAM (1990) Recent advances in thin films for magneto-optic recording. Vacuum 41: 1160-1165.

12. Zharov AA, Kurina VV (2007) Giant resonant magneto-optic Kerr effect in nanostructured ferromagnetic metamaterials J Appl Phys 102: 123514-4.

13. Bennett LH, Della Torre E (2014) Bose-Einstein Condensation of Confined Magnons in Nanostructures. J Mod Phys 5: 693-705.

14. Xia J, Beyersdorf PT, Fejer MM, Kapitulnik A (2006) Modified Sagnac interferometer for high-sensitivity magneto-optic measurements at cryogenic temperatures. Appl Phys Lett 89: 062508-3.

15. Li J, Przybylski M, Yildiz F, Ma XD, Wu YZ (2009) Oscillatory Magnetic Anisotropy Originating from Quantum Well States in Fe Films. Phys Rev Lett 102: 207206-4.

16. Bauer U, Przybylski M (2010) Large amplitude oscillation of magnetic anisotropy engineered by substrate step density. Phys Rev B 81: 134428-6.

17. Li J, Przybylski M, He Y, Wu YZ (2010) Experimental observation of quantum oscillations of perpendicular anisotropy in Fe films on $\mathrm{Ag}(1,1,10)$. Phys Rev B 82: 214406-6.

18. Bauer U, Dąbrowski M, Przybylski M, Kirschner J (2011) Experimental confirmation of quantum oscillations of magnetic anisotropy in $\mathrm{Co} / \mathrm{Cu}(001)$. Phys Rev B 84: 144433-5.

19. Bauer U, Przybylski M, Beach GSD (2014) Voltage control of magnetic anisotropy in Fe films with quantum well states. Phys Rev B 89: 174402-7.

20. Andrews SS, Boxer SG (2000) A liquid nitrogen immersion cryostat for optical measurements. Rev Sci Instrum 71: 3567-3569.

21. Qiu ZQ, Bader SD (2000) Surface magneto-optic Kerr effect. Rev Sci Instrum 71: $1243-1255$

22. Edwards MH (1956) The Index of Refraction of Liquid Helium. Can J Phys 34: $898-900$

23. Radebaugh $R$ (2009) Cryocoolers: the state of the art and recent developments. J Phys: Condens Matter 21: 164219-9.

24. Sharrock MP (1994) Time dependence of switching fields in magnetic recording media. J Appl Phys 76: 6413-6418. 